- Emphasises the benefits of correct seating and good posture.

- May benefit dental students and dentists in introducing the concept of healthy working posture.

- Introduces the relationship between working posture and musculoskeletal disorders.

\title{
Assessment of dental student posture in two seating conditions using RULA methodology - a pilot study
}

\author{
A. Gandavadi, ${ }^{1}$ J. R. E. Ramsay ${ }^{2}$ and F. J. T. Burke ${ }^{3}$
}

\begin{abstract}
Objectives To assess dental students' posture on two different seats in order to determine if one seat predisposes to a difference in working posture. Design A between-subject experimental design was selected. Setting The study was undertaken at the University of Birmingham School of Dentistry in 2006. Subjects (materials) and methods Sixty second year dental students at the University of Birmingham who were attending their first classes in the phantom head laboratory were randomly selected and allocated to two different seats (30 Bambach Saddle Seats and 30 conventional seats). Students were trained in the use of the seats. After ten weeks, the students were observed, photographs were taken by the researcher and these were assessed using Rapid Upper Limb Assessment (RULA). Main outcome measures The posture of the students was assessed using the RULA. Each student was given a risk score. A Mann Whitney test was used for statistical analysis. Results The results indicated that the students using the conventional seat recorded significantly higher risk scores $(p<0.05)$ when compared with the students using Bambach Saddle Seat, suggesting an improvement in posture when using the Bambach Saddle Seat. Conclusion RULA has identified that dental students using a Bambach Saddle Seat were able to maintain an acceptable working posture during simulated dental treatment and this seating may reduce the development of work-related musculoskeletal disorders.
\end{abstract}

\section{INTRODUCTION AND BACKGROUND}

In recent times, dentistry has been considered to be a demanding profession due to the need for high concentration and precision. ${ }^{1}$ Work-related musculoskeletal disorders, especially of the neck and upper limbs, have become common among dentists, ${ }^{2-7}$ most notably

\footnotetext{
${ }^{*}$ PhD student, ${ }^{2}$ Lecturer in Physiotherapy, University of Birmingham, School of Health Sciences, 52 Pritchatts Road, University of Birmingham, Edgbaston, Birmingham, B15 2TT; ${ }^{3}$ Professor of Primary Dental Care, The School of Dentistry, University of Birmingham, St Chad's Queensway

Birmingham, B4 6NN

*Correspondence to: Mr Amar Gandavadi

Email:axg284@bham.ac.uk
}

\section{Refereed Paper}

Accepted 12 July 2007

DOI: $10.1038 /$ bdj.2007.1047

${ }^{\circ}$ British Dental Journal 2007; 203: 601-605 with low back pain. ${ }^{8}$ Such injuries may lead to premature retirement. ${ }^{9}$ Current dental practice has the dentist working in a sitting position at one side of the patient, who is in a supine position. ${ }^{1}$ The dentist will also generally have a chairside assistant sitting at the other side of the patient. ${ }^{4}$ Because of the restricted work area (the mouth), and the need for dexterity, the dentist may need to adopt inflexible work postures. This results in static activity of the muscles in awkward postures and may lead to musculoskeletal disorders. For example, Lake ${ }^{10}$ reported that dentists who qualified in Canada spent approximately two-thirds of each treatment hour in 19-54 degrees of forward trunk inclination, which increased disc pressures considerably, ${ }^{11}$ when compared to standing.
The aim of this study was to investigate whether modification of seating improves posture in dental students.

\section{Sitting posture}

Grandjean ${ }^{12}$ describes sitting as 'a natural human posture' because it relieves the person of the need to actively maintain an upright posture. This reduces the static muscular workload required to maintain the joints of the foot, knee, hip and spine, with a consequent reduction in energy consumption. Seating may improve stability and performance in tasks that require fine or precise upper limb movements. Sitting reduces the weight bearing on the lower limbs, allowing an improved posture for foot control operations. However, sitting in a slumped posture may contribute to the 


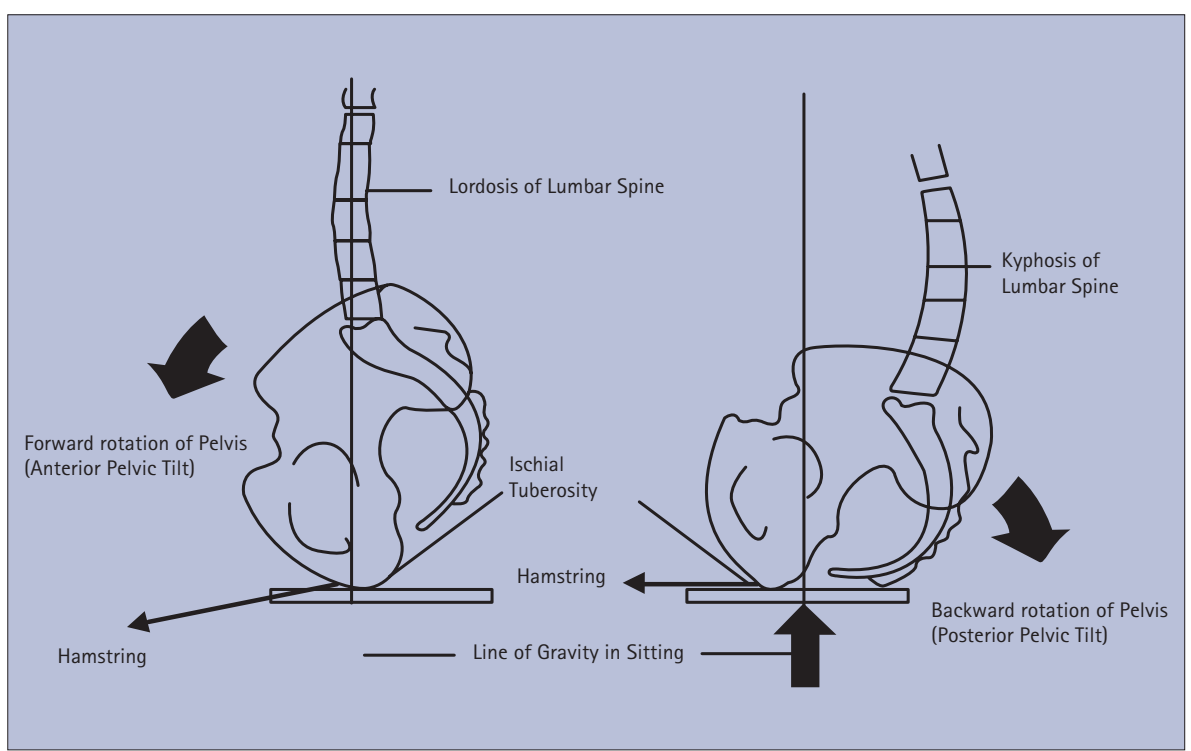

Fig. 1 Sitting posture (anterior and posterior pelvic tilt)

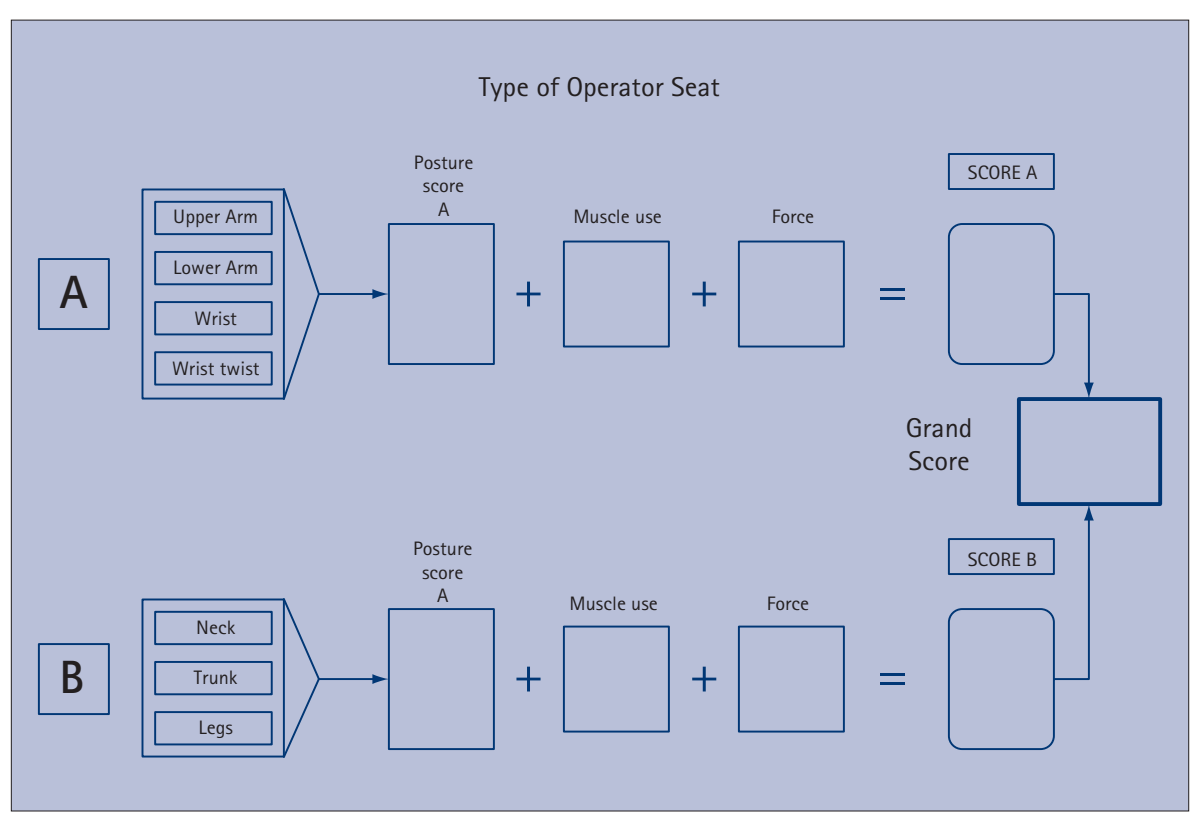

Fig. 2 RULA scoring sheet (adapted from McAtamney and Corlett ${ }^{22}$ )

development of musculoskeletal disorders eg low back pain and neck pain. ${ }^{13}$ Prolonged sitting may slacken the abdominal muscles and make the spine slump. ${ }^{13,14}$ This, in turn, increases the strain of spinal ligaments and stretches the back muscles. ${ }^{13}$ If this posture is adopted over time, fatigue and back pain may be reported. ${ }^{15,16}$ This slumped posture also increases vertebral disc pressure, again presenting as back pain ${ }^{14}$ and may eventually lead to disc herniation. ${ }^{17}$ This back pain may also impair the activation of transversus abdominis muscles $^{18}$ and oblique abdominal muscles. ${ }^{19}$ These muscles are responsible for stabilising the spine and for postural control of the trunk during seated postures; ${ }^{18}$ the postural activation of these muscles may tension in the hamstring muscles alters when the angle of knee flexion and hip flexion varies in sitting. When a person is seated with the hips at 60 degrees from the vertical with a forward sloping seat, the tension in the hamstring muscles are relieved and the pelvis is pulled forward. This, in turn, results in lordosis of the lumbar spine (anterior pelvic tilt; Fig. 1). Pynt ${ }^{21}$ recommended lumbar lordosed seated posture, regularly interspersed with movement (lordosis to kyphosis) as the optimal sitting posture, which is necessary to maintain lumbar postural health, and the prevention of low back pain. The Bambach Saddle Seat (BSS) is designed to facilitate this position.

\section{METHODOLOGY}

\section{Research design}

A between-subject experimental design was selected. The postures in two different seats with different subjects performing the same dental procedure were compared. The working posture adopted by each student was evaluated using Rapid Upper Limb Assessment ${ }^{22}$ (RULA; Fig. 2). RULA is a survey method originally developed to assess posture in ergonomic investigations in workplaces where work-related upper limb disorders are reported, such as in Visual Display Unit (VDU) operators and operators working in a variety of manufacturing tasks. ${ }^{22}$ Since dentists work in a prolonged sitting posture, which resembles some manufacturing tasks, it could be considered that RULA would be a useful tool in assessing the working posture of dentists.

\section{Ethics}

The research was approved by the School of Health Sciences, University of Birmingham ethics committee. tures are adopted. It has been found that the activity of the oblique abdominal muscles is significantly higher in standing and seated postures when compared to lying, suggesting an important role in sustaining gravity loads ${ }^{19}$ and stabilising these postures.

Pheasant ${ }^{20}$ argued that the action of sitting down on a seat of 'average' height involves flexion of the knees and hips to about 90 degrees in each. The comfortable limit of hip joint flexion is about 60 degrees from the vertical, beyond which the passive tension of the hamstring muscles increases, which, in turn, pulls the pelvis backwards to about 30 degrees. This results in kyphosis of the lumbar spine (posterior pelvic tilt; Fig. 1). The

\section{Participants}

The aim and nature of the study was introduced to all of the Year 2 dental students at the Dental School who were attending their first classes in the phantom head laboratory. Ninety students were provided with information sheets and consent forms. The students were asked to return the forms if they were willing to participate in the study. Sixty students were selected at random from the $80 \mathrm{stu}$ dents who returned the forms and agreed to participate in the study. The students were randomly selected using a random number generator ${ }^{23}$ and allocated to two 
types of seats. Thirty students were provided with Bambach Saddle Seat (BSS) and 30 students were provided with the conventional seats (CS).

\section{Training}

The students attended a lecture on the use of seats before commencement of the study. The students were followed up during the first two weeks on their sessions in the phantom head lab and individually trained for five to 10 minutes on correct operating posture respective to their seats.

\section{Materials}

- BSS (Fig. 3)

- CS (Fig. 4)

- Phantom Head Apparatus (Figs 3-4)

- Digital Camera (Nikon Coolpix 8400, Tokyo).

\section{Assessment procedure}

After ten weeks, photographs were taken of the students whilst working in the phantom head lab. The positions recorded on the photographs were assessed using RULA.

The guidelines considered for taking photographs were:

- Photographs were used to allow analysis of the student's posture at a later date. Photographs of the student were taken from all possible angles in order to enable viewing of all joints to be analysed

- Photographs were taken ten minutes after the student had started the dental procedure, which was considered sufficient to allow time for the student to become comfortable in their operating position.

\section{RULA}

The RULA method uses diagrams of body postures and three scoring tables to provide evaluation of exposure to risk factors by providing a risk score. The RULA scoring sheet is schematically shown in Figure 2. According to RULA, the body is divided into two segments ( $\mathrm{A}$ and $\mathrm{B}$ ) and assessed:

A. Upper arm, lower arm and wrist

B. Neck, trunk and legs.

The range of movement for each body part is divided into segments and recorded appropriately. The minimum score (Score 1) is accorded to the ranges of movement where the risk factors are minimal and higher numbers (up to 6) are given to ranges of movement with extreme postures. An individual posture score (Score A and B calculated from separate tables) is given to each body segment (Segments A and B) during analysis and these scores, along with muscle use score (muscle use scores are estimated for static postures held for longer than one minute or repeated more than four times per minute), and force scores (force score is estimated for total hours of work in a day), these are then read from a separate table to reveal the grand score (risk score).

The risk score ranges from 1 to 7 in which a score of 1 or 2 is acceptable, a score of 3 or 4 needs further investigation, 5 or 6 needs investigation and changes sooner and a score of 7 needs immediate investigation and change.

\section{Modified RULA suitable for dentists}

The original RULA was developed to assess only one side of the body at a particular time for the selected work posture. To assess the other side of the body the assessment has to be repeated and the selected work posture to be assessed may have changed. To avoid this difficulty and to save time the RULA recording chart was modified and designed to record details of both sides of the body at the same time. A separate RULA score (risk score) for the right and left side was ascribed to each posture assessed. ${ }^{24}$

\section{The dental procedure}

Photographs were taken when the students were operating on teeth in the lower jaw of the phantom head whilst preparing the teeth for a restoration. Figures 3 and 4 show a dental student in two different seats.

\section{Analysis of photographs}

The photographs of 60 students (30 students using the BSS and 30 students using the CS) were analysed by the researcher using the modified RULA described above. Each student was given a risk score, which was used for statistical analysis.

\section{Data analysis}

The hypotheses are two tailed. A MannWhitney Test was used to test the hypothesis that there will be a difference between the RULA scores achieved between the students using the BSS and CS. The level

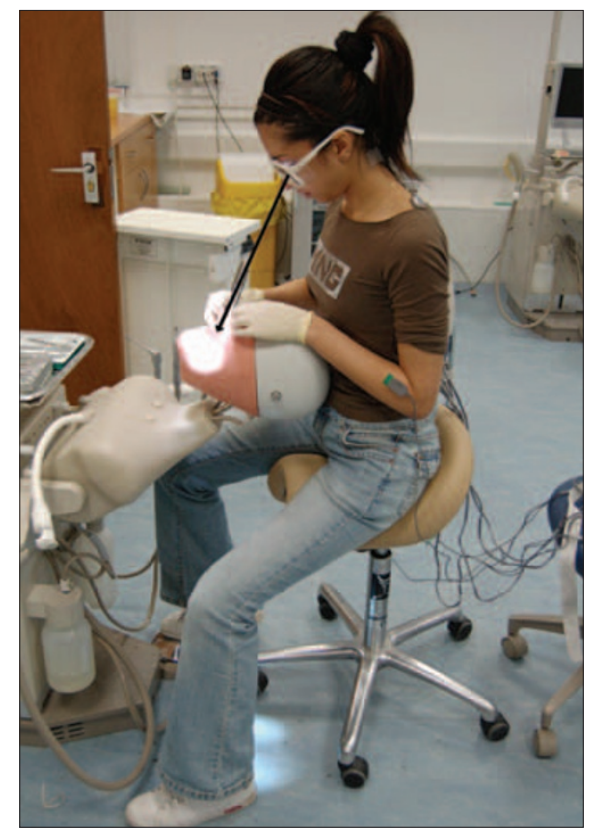

Fig. 3 A dental student working on a Bambach Saddle Seat (reproduced with permission from Bambach)

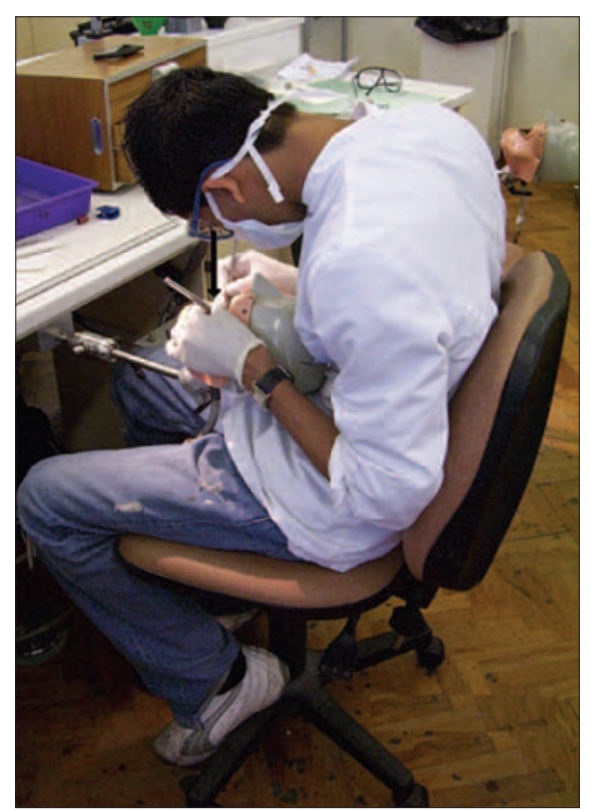

Fig. 4 A dental student working on a conventional seat

of significance of 0.05 was used for the rejection of the null hypothesis.

\section{RESULTS}

The photographs of 60 students were analysed using RULA. Figures 5 and 6 show the mean and standard errors for the right and left total scores comparing BSS and CS. The Mann-Whitney results were significant $(\mathrm{Z}=-6.015 ; p<0.01)$ for the right total score and $(\mathrm{Z}=-6.197$; $p<0.01$ ) for the left total score. The results confirmed that there was a significant difference in RULA scores between the 
seats. Thus the null hypothesis can be rejected and the experimental hypothesis can be accepted. The results also indicated that the students using the CS recorded significantly higher RULA scores $($ mean $=5.06$ for the right side; mean $=5.03$ for the left side) when compared with the students using the BSS ( mean $=2.80$ for the right side; mean $=$ 2.66 for the left side) ( $p<0.01)$, suggesting that there is a lower postural risk when using the Bambach Saddle Seat. The eta ${ }^{2}$ has been calculated to obtain the effect size, the eta ${ }^{2}$ for right total score is 0.43 and eta ${ }^{2}$ for left total score is 0.45 which indicates large effect size.

\section{DISCUSSION}

The results indicate that there is a statistically significant difference between the risk scores of the BSS and the CS. The students using the BSS were able to maintain an acceptable position on the observed joints (upper limb, trunk and lower limb), which may be considered to contribute to a healthy working posture. The students using the CS appeared less able to maintain a healthy posture with the observed joint positions, indicating a cause for concern. The results indicate that the standard deviation of the risk scores (right and left grand scores) for the students using the BSS were negligible when compared with the CS. However, the left grand score for the students using the BSS had indicated a standard deviation of 0.47 on risk scores. This may be because most of the students operate with the right hand and showed an acceptable risk score on the right side, whereas their left hand was kept at a position of reduced risk, close to the body with joints in a safe range, thereby decreasing the final risk score from 3 to 2 . There was variation in the position of the left hand, with some students holding the cheek of the phantom head in order to get an improved vision of the teeth which were being operated on, while others used a mirror or rested their left hand on their thigh. The students using the CS recorded higher risk scores (mean of 5) with a standard deviation of 1.36 on both the sides observed, indicating poor posture. Even though the position of their left hand was similar to that of the students using the BSS, their slumped posture kept their joints at extreme ranges ie their shoulders were kept elevated and abducted with

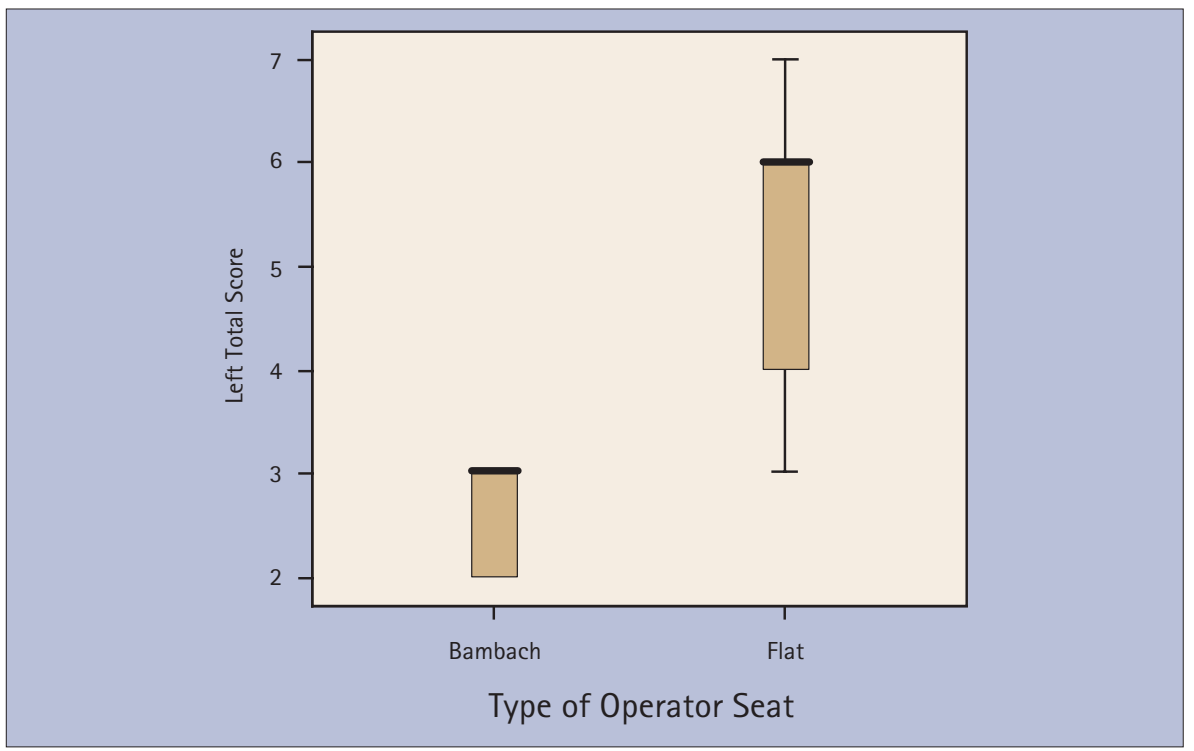

Fig. 5 Box plot showing the left grand score

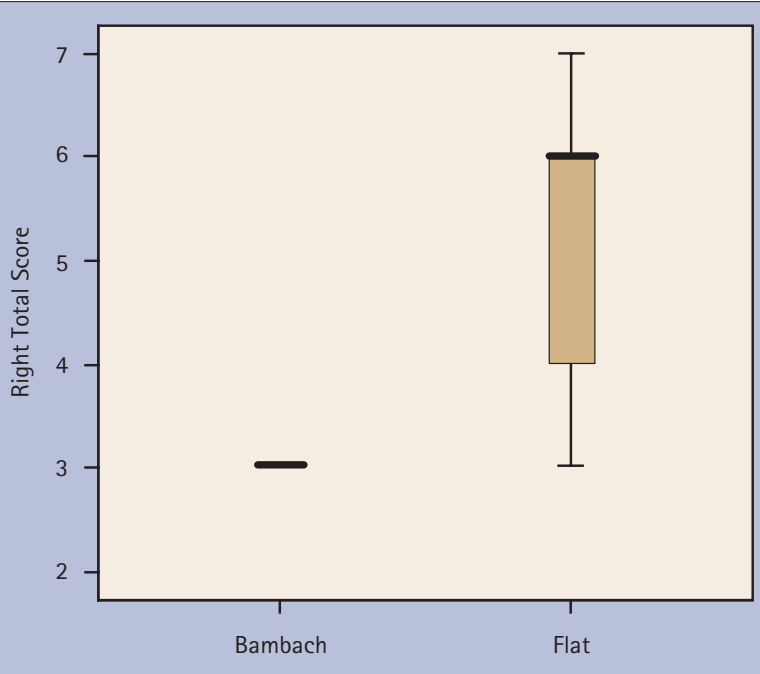

Type of Operator Seat

Fig. 6 Box plot showing the right grand score

their arm working across the midline of their body, thereby increasing the range of their final risk scores ranging from 3 to 7 indicating extreme concern which requires immediate investigation and changes.

The spine is in its natural curved position ('S' shape) while standing, enabling the body's line of gravity to pass through the trunk and feet, so requiring minimal muscular activity to maintain the posture and to hold the trunk erect. ${ }^{25}$ Callaghan and McGill ${ }^{26}$ found that standing produced a uniquely different spine posture compared with sitting, and standing spine postures did not overlap with flexion postures adopted in sitting. Sitting with a 90 degree angle between the trunk and the thighs causes the pelvis to rotate backwards shifting the spine away from the line of gravity (Fig. 1). This in turn reduces the lumbar lordosis, ${ }^{12}$ causing the spine to slump and increasing the load placed on the spine. ${ }^{27}$ Black et al. ${ }^{28}$ found that the movement of the lumbar spine influenced the movement of the cervical spine and identified the slumped sitting posture (posterior pelvic tilt) as the poor posture for the spine. The students using the CS may have registered higher risk scores as a result of sitting in posterior pelvic tilt (kyphosis of the lumbar spine). They recorded higher risk scores in the neck (hyper flexion), shoulder (raised and abducted) and trunk ${ }^{10}$ (slumped/forward inclined) (Fig. 4) since the position of neck, shoulders and trunk are interrelated and an acceptable spinal posture is necessary to maintain good sitting 
posture. ${ }^{27,28}$ On the other hand, the students using the BSS were able to maintain an acceptable position of the neck, shoulders and trunk, as they were able to maintain anterior pelvic tilted position contributing to the lower risk score (Fig. 3). The BSS is designed to maintain the pelvis in an anterior tilted position in order to achieve a slight lumbar lordosis $^{29}$ and the angle of hips and knees can be adjusted so that the spinal posture simulates standing, thereby contributing to a healthy spinal posture.

\section{CONCLUSION}

The RULA method applied to dental students' working postures allowed a rapid evaluation of their posture during simulated dental treatment. The RULA scores indicate that the students using the BSS are able to maintain an acceptable working posture (lower risk score), whereas for students using the CS the posture deteriorates over time (higher risk score). This may predispose to the development of musculoskeletal disorders. This study serves as a pilot trial and further studies in other dental schools and with practising dentists is suggested.

This project was funded by Bambach Saddle Seat Europe Ltd and undertaken independently at the University of Birmingham. We are grateful to all of the students who participated in this study and also the staff members at the University of Birmingham who assisted in this study.
1. Finsen L, Christensen H, Bakke M. Musculoskeletal disorders among dentists and variation in dental work. Appl Ergon 1998; 29: 119-125.

2. Milerad E, Ekenvall L. Symptoms of the neck and upper extremities in dentists. Scand J Work Environ Health 1990; 16: 129-134.

3. Osborn J, Newell K, Rudney J, Stoltenberg J. Musculoskeletal pain among Minnesota dental hygienists. J Dent Hyg 1990; 64: 132-138.

4. Rundcrantz B-L, Johnsson B, Moritz U. Cervical pain and discomfort among dentists. Epidemiological, clinical and therapeutic aspects. Part 1. A survey of pain and discomfort. Swed Dent J 1990; 14: 71-80.

5. Oberg T, Oberg U. Musculoskeletal complaints in dental hygiene: a survey study from a Swedish country. J Dent Hyg 1993; 67: 257-261.

6. Akesson I, Lundborg G, Horstmann V, Skerfving S. Neuropathy in female dental personnel exposed to high frequency vibrations. Occup Environ Med 1995; 52: 116-123.

7. Finsen L. Biomechanical analyses of occupational work loads in the neck and shoulder. A study in dentistry. PhD thesis, National Institute of Occupational Health, Copenhagen, Human Physiology, August Krogh Institute at University of Copenhagen, Department of Oral Function and Physiology, School of Dentistry, 1995.

8. Shugars D, Miller D, Williams D, Fishburne $C$, Strickland D. Musculoskeletal pain among general dentists. Gen Dent 1987; 35: 272-276.

9. Burke F J T, Freeman R. The practice of dentistry: an assessment of reasons for premature retirement. Br Dent J 1997; 182: 250-254.

10. Lake J. Musculoskeletal dysfunction associated with the practice of dentistry - proposed mechanisms and management: literature review. Univ Tor Dent J 1995; 9: 7-11.

11. Nachemson A. The lumbar spine: an orthopedic challenge. Spine 1976; 1.

12. Grandjean E. Ergonomics in the home. London: Taylor and Francis, 1973.

13. Beach T A C, Parkinson R J, Stothart J, Callaghan J P. Effects of prolonged sitting on passive stiffness of the in vivo lumbar spine. Spine J 2005; 5: 145-154.

14. McGill S M, Brown S. Creep response of the lumbar spine to prolonged flexion. Clin Biomech 1992; 7: 43-46.

15. Mannion A F. Fibre type characteristics and func- tion of the human paraspinal muscles: normal values and changes in association with low back pain. J Electromyogr Kinesiol 1999; 9: 363-377.

16. Gunning J, Callaghan J P, McGill S M. The role of prior loading history and spinal posture on the compressive tolerance and type of failure in the spine using a porcine trauma model. Clin Biomech 2001; 16: 471-480.

17. Bogduk N. Clinical anatomy of the lumbar spine and sacrum, $4^{\text {th }}$ ed. Edinburgh: Churchill Livingstone, 2005.

18. Moseley G L. Impaired trunk muscle function in sub-acute neck pain: etiologic in the subsequent development of low back pain? Man Ther 2004; 9: 157-163.

19. Snijders C J, Bakker M P, Vleeming A, Stoeckart R, Stam $\mathrm{H}$ J. Oblique abdominal muscle activity in standing and in sitting on hard and soft seats. Clin Biomech 1995; 10: 73-78.

20. Pheasant S. Ergonomics, work and health. UK: Palgrave Macmillan, 1991.

21. Pynt J, Higgs J, Mackey M. Seeking the optimal posture of the seated lumbar spine. Physiother Theory Pract 2001; 17: 5-21.

22. McAtamney L, Corlett E N. RULA: a survey method for the investigation of work-related musculoskeletal disorders. Appl Ergon 1993; 24: 91-99.

23. Random Number Generator. http://www segobit. com/rng.htm. Accessed 27 July 2006.

24. Nguyen B D. Musculoskeletal problems and occupational stress among dentists and dental assistants in a dental clinic. National Institute of Occupational and Environmental Health, Vietnam. Occup Environ Med 2004; 61: e57. http://www.occenvmed.com/ cgi/content/full/61/11/e57. Accessed 27 July 2006.

25. Corlett E N, Eklund J A E. How does a back rest work? Appl Ergon 1984; 15: 111-114.

26. Callaghan J P, McGill S M. Low back joint loading and kinematics during standing and unsupported sitting. Ergonomics 2001; 44: 280-294.

27 Hedman TP, Fernie G R. Mechanical response of the lumbar spine to seated postural loads. Spine 1997; 22: 734-743.

28. Black K M, McClure P, Polansky M. The influence of different sitting positions on cervical and lumbar posture. Spine 1996; 21: 65-70.

29. Gale M, Feather S, Jensen S, Coster G. Study of a workseat designed to preserve lumbar lordosis. Aust Occup Ther J 1989; 36: 92-99. 